\title{
Analytical properties of the Hurwitz-Lerch zeta function
}

\author{
Raghib Nadeem', Talha Usman², Kottakkaran Sooppy Nisar ${ }^{3^{*}}$ (D) and Dumitru Baleanu 4,5,6
}

\author{
"Correspondence: \\ n.sooppy@psau.edu.sa \\ ${ }^{3}$ Department of Mathematics, \\ College of Arts and Sciences, Prince \\ Sattam bin Abdulaziz University, \\ Wadi Aldawaser, 11991, Saudi \\ Arabia \\ Full list of author information is \\ available at the end of the article
}

\begin{abstract}
In the present paper, we aim to extend the Hurwitz-Lerch zeta function $\Phi_{\delta, 5 ; \gamma}(\xi, s, v ; p)$ involving the extension of the beta function (Choi et al. in Honam Math. J. 36(2):357-385, 2014). We also study the basic properties of this extended Hurwitz-Lerch zeta function which comprises various integral formulas, a derivative formula, the Mellin transform, and the generating relation. The fractional kinetic equation for an extended Hurwitz-Lerch zeta function is also obtained from an application point of view. Furthermore, we obtain certain interesting relations in the form of particular cases.
\end{abstract}

MSC: $33 C 05 ; 33 C 45 ; 33 C 47 ; 33 C 90$

Keywords: Generalized; Generating functions; Rodrigues formula

\section{Overture}

The familiar Hurwitz-Lerch zeta function $\Phi(\xi, s, v)$ is defined by (see, e.g., [2, p. 27, Sect. 1.11, Eq. (1)]; see also [3])

$$
\begin{aligned}
\Phi(\xi, s, v) & =\sum_{m=0}^{\infty} \frac{\xi^{m}}{(m+v)^{s}} \\
& (v \neq\{0,-1,-2, \ldots\} ; s \in \mathbb{C} \text { when }|\xi|<1 ; \Re(s)>1 \text { when }|\xi|=1) .
\end{aligned}
$$

A more detailed exposition of the various generalizations, properties, and applications of the Hurwitz-Lerch zeta functions could be found in the literature (see [3-12]). For example Goyal and Laddha [9], Lin and Srivastava [13] and Garg et al. [7] established certain remarkable extensions of the Hurwitz-Lerch zeta function $\Phi_{\delta, 5 ; \gamma}(\xi, s, v)$ given in Eq. (1.1), which are described, respectively, by

$$
\begin{aligned}
& \Phi_{\delta}^{*}(\xi, s, v)=\sum_{m=0}^{\infty} \frac{(\delta)_{m}}{m !} \frac{\xi^{m}}{(m+v)^{s}} \\
&(\delta \in \mathbb{C} ; v \neq\{0,-1,-2, \ldots\}, s \in \mathbb{C} \text { when }|\xi|<1 ; \Re(s-\delta)>1 \text { when }|\xi|=1), \\
& \Phi_{\zeta, \gamma}^{(\eta, \omega)}(\xi, s, v)=\sum_{m=0}^{\infty} \frac{(\zeta)_{\eta m}}{(\gamma)_{\omega m}} \frac{\xi^{m}}{(m+v)^{s}}
\end{aligned}
$$

(c) The Author(s) 2020. This article is licensed under a Creative Commons Attribution 4.0 International License, which permits use, sharing, adaptation, distribution and reproduction in any medium or format, as long as you give appropriate credit to the original author(s) and the source, provide a link to the Creative Commons licence, and indicate if changes were made. The images or other third party material in this article are included in the article's Creative Commons licence, unless indicated otherwise in a credit line to the material. If material is not included in the article's Creative Commons licence and your intended use is not permitted by statutory regulation or exceeds the permitted use, you will need to obtain permission directly from the copyright holder. To view a copy of this licence, visit http://creativecommons.org/licenses/by/4.0/. 


$$
\begin{aligned}
& \left(\varsigma \in \mathbb{C} ; v, \gamma \neq\{0,-1,-2, \ldots\} ; \eta, \omega \in \mathbb{R}^{+} ; \eta<\omega \text { when } s, \xi \in \mathbb{C}\right. \\
& \eta=\omega \text { and } s \in \mathbb{C} \text { when }|\xi|<1, \eta=\omega \text { and } \Re(s-\varsigma+\gamma)>1 \text { when }|\xi|=1)
\end{aligned}
$$

and

$$
\begin{aligned}
& \Phi_{\delta, \varsigma ; \gamma}(\xi, s, v)=\sum_{m=0}^{\infty} \frac{(\delta)_{m}(\varsigma)_{m}}{(\gamma)_{m}(m+v)^{s}} \frac{\xi^{m}}{m !} \\
& (\delta, \varsigma \in \mathbb{C} ; \gamma, v \neq\{0,-1,-2, \ldots\} ; s \in \mathbb{C} \text { when }|\xi|<1 ; \\
& \Re(s+\gamma-\delta-\varsigma)>1 \text { when }|\xi|=1),
\end{aligned}
$$

where $(\delta)_{m}$ stands for the Pochhammer symbol (for $\delta \in \mathbb{C}$ ) given by (see [14, p. 22, Eq. (1)])

$$
(\delta)_{m}=\frac{\Gamma(\delta+m)}{\Gamma(\delta)}= \begin{cases}\delta(\delta+1)(\delta+2) \cdots(\delta+m-1) & (m \geqq 1, \delta \in \mathbb{C}), \\ 1 & (m=0, \delta \in \mathbb{C} \backslash\{0\}) .\end{cases}
$$

The integral representation of Eqs. (1.2), (1.3) and (1.4) are given below, respectively,

$$
\begin{array}{r}
\Phi_{\delta}^{*}(\xi, s, v)=\frac{1}{\Gamma(s)} \int_{0}^{\infty} \frac{t^{s-1} e^{-v t}}{\left(1-\xi e^{-t}\right)^{\delta}} d t=\frac{1}{\Gamma(s)} \int_{0}^{\infty} \frac{t^{s-1} e^{-(v-1) t}}{\left(e^{t}-\xi\right)^{\delta}} d t \\
(\Re(v)>0 ; \Re(s)>0 \text { when }|\xi| \leqq 1(\xi \neq 1) ; \Re(s)>1 \text { when } \xi=1), \\
\Phi_{5, \gamma}^{(\eta, \omega)}(\xi, s, v)=\frac{1}{\Gamma(s)} \int_{0}^{\infty} t^{s-1} e^{-v t}{ }_{2} \psi_{1}\left[\begin{array}{l}
(\varsigma ; \eta),(1 ; 1) ; \xi e^{-t} \\
(\gamma ; \omega)
\end{array}\right] d t \\
(\Re(v)>0 ; \Re(s)>0 \text { when }|\xi| \leqq 1(\xi \neq 1) ; \Re(s)>1 \text { when } \xi=1),
\end{array}
$$

where ${ }_{2} \psi_{1}$ is the Fox-Wright function defined in [8] and

$$
\begin{aligned}
& \Phi_{\delta, \zeta ; \gamma}(\xi, s, v)=\frac{1}{\Gamma(s)} \int_{0}^{\infty} t^{s-1} e^{-v t}{ }_{2} F_{1}\left(\delta, \varsigma ; \gamma ; \xi e^{-t}\right) d t \\
& (\Re(v)>0 ; \Re(s)>0 \text { when }|\xi| \leqq 1(\xi \neq 1) ; \Re(s)>1 \text { when } \xi=1) .
\end{aligned}
$$

Firstly, in 2014 Parmar and Raina [15] introduced the generalized Hurwitz-Lerch zeta function involving the extended beta function [16] given by

$$
\begin{aligned}
& \Phi_{\delta, \varsigma ; \gamma}(\xi, s, v ; p)=\sum_{m=0}^{\infty} \frac{(\delta)_{m}}{m !} \frac{B_{p}(\varsigma+m, \gamma-\varsigma)}{B(\varsigma, \gamma-\varsigma)} \frac{\xi^{m}}{(m+v)^{s}} \\
& (p \geqq 0 ; \delta, \varsigma \in \mathbb{C} ; \gamma, v \neq\{0,-1,-2, \ldots\} ; s \in \mathbb{C} \text { when }|\xi|<1 ; \\
& \Re(s+\gamma-\delta-\varsigma)>1 \text { when } \xi=1),
\end{aligned}
$$

and they also established their integral representation as follows:

$$
\begin{aligned}
& \Phi_{\delta, \varsigma ; \gamma}(\xi, s, v ; p)=\frac{1}{\Gamma(s)} \int_{0}^{\infty} t^{s-1} e^{-v t} F_{p}\left(\delta, \varsigma ; \gamma ; \xi e^{-t}\right) d t \\
& (p \geqq 0 ; \Re(v)>0 ; \Re(s)>0 \text { when }|\xi| \leqq 1(\xi \neq 1) ; \Re(s)>1 \text { when } \xi=1),
\end{aligned}
$$


where the extended beta function $B\left(\delta_{1}, \delta_{2} ; p\right)$ appears in (1.8) and extended hypergeometric function $F_{p}(a, b ; c ; \xi)$ [17] appears in (1.9) defined, respectively, by

$$
\begin{gathered}
B\left(\delta_{1}, \delta_{2} ; p\right)=\int_{0}^{1} t^{\delta_{1}-1}(1-t)^{\delta_{2}-1} \exp \left(-\frac{p}{t(1-t)}\right) d t \\
\left(\Re(p)>0, \Re\left(\delta_{1}\right)>0, \Re\left(\delta_{2}\right)>0\right),
\end{gathered}
$$

and

$$
\begin{gathered}
F_{p}(a, b ; c ; \xi)=\sum_{m=0}^{\infty}(a)_{m} \frac{B_{p}(b+m, c-b)}{B(b, c-b)} \frac{(\xi)^{m}}{m !} \\
(p \geqq 0,|\xi|<1 ; \Re(c)>\Re(b)>0) .
\end{gathered}
$$

Obviously for $p=0$, Eq. (1.8) reduces directly to (1.4).

Moreover, Choi et al. [1] established the underlying generalization of extended beta and extended hypergeometric functions given by, respectively,

$$
\begin{gathered}
B_{p, q}\left(\delta_{1}, \delta_{2}\right)=\int_{0}^{1} t^{\delta_{1}-1}(1-t)^{\delta_{2}-1} \exp \left(-\frac{p}{t}-\frac{q}{1-t}\right) d t \\
\left(\Re(p)>0 ; \Re(q)>0 ; \Re\left(\delta_{1}\right)>0 ; \Re\left(\delta_{2}\right)>0\right),
\end{gathered}
$$

and

$$
\begin{gathered}
F_{p, q}(a, b ; c ; \xi)=\sum_{m=0}^{\infty}(a)_{m} \frac{B_{p, q}(b+m, c-b)}{B(b, c-b)} \frac{(\xi)^{m}}{m !} \\
(p \geqq 0, q \geqq 0,|\xi|<1 ; \Re(c)>\Re(b)>0) .
\end{gathered}
$$

It is clearly seen that Eqs. (1.10) and (1.11) are particular cases of Eq. (1.12) and Eq. (1.13), respectively, provided when $p=q$.

Motivated by those various fascinating extensions of Hurwitz-Lerch zeta function, further we establish an extension of generalized Hurwitz-Lerch zeta function involving extended beta function $B\left(\delta_{1}, \delta_{2} ; p, q\right)$.

\section{A new extension of the Hurwitz-Lerch zeta function}

In this section, we consider a new extension of the generalized Hurwitz-Lerch zeta function involving extended beta function [1] given by

$$
\begin{aligned}
& \Phi_{\delta, \varsigma ; \gamma}(\xi, s, v ; p, q)=\sum_{m=0}^{\infty} \frac{(\delta)_{m}}{m !} \frac{B_{p, q}(\varsigma+m, \gamma-\varsigma)}{B(\varsigma, \gamma-\varsigma)} \frac{\xi^{m}}{(m+v)^{s}} \\
& (p \geq 0, q \geq 0 ; \delta, \varsigma \in \mathbb{C} ; \gamma, v \neq\{0,-1,-2, \ldots\} ; s \in \mathbb{C} \text { when }|\xi|<1 \\
& \Re(s+\gamma-\delta-\varsigma)>1 \text { when }|\xi|=1) .
\end{aligned}
$$

Remark 2.1 We enumerate the following particular and limiting cases of the function $\Phi_{\delta, 5, \gamma}(\xi, s, v ; p, q)$ : 
(i) In Eq. (2.1) substituting $\delta=1$, we obtain a new extended form of the generalized Hurwitz-Lerch zeta function found by Lin and Srivastava [13]:

$$
\begin{aligned}
& \Phi_{\varsigma, \gamma}^{1,1}(\xi, s, v ; p, q)=\Phi_{1, \varsigma, \gamma}(\xi, s, v ; p, q)=\sum_{m=0}^{\infty} \frac{B_{p, q}(\varsigma+m, \gamma-\varsigma)}{B(\varsigma, \gamma-\varsigma)} \frac{\xi^{m}}{(m+v)^{s}} \\
& (p \geq 0, q \geq 0 ; \varsigma \in \mathbb{C} ; \gamma, v \neq\{0,-1,-2, \ldots\} ; s \in \mathbb{C} \text { when }|\xi|<1 ; \\
& \Re(s+\gamma-\varsigma)>1 \text { when }|\xi|=1) .
\end{aligned}
$$

(ii) If we set $q=1$, Eq. (2.2) reduces the extended Hurwitz-Lerch zeta function introduced in [15, p. 160, Eq. (2.2)]:

$$
\begin{aligned}
& \Phi_{\varsigma, \gamma}^{1,1}(\xi, s, v ; p)=\Phi_{1, \varsigma, \gamma}(\xi, s, v ; p)=\sum_{m=0}^{\infty} \frac{(\delta)_{m}}{m !} \frac{B_{p}(\varsigma+m, \gamma-\varsigma)}{B(\varsigma, \gamma-\varsigma)} \frac{\xi^{m}}{(m+v)^{s}} \\
& (p \geq 0, \varsigma \in \mathbb{C} ; \gamma, v \neq\{0,-1,-2, \ldots\} ; s \in \mathbb{C} \text { when }|\xi|<1 ; \\
& \Re(s+\gamma-\varsigma)>1 \text { when }|\xi|=1) .
\end{aligned}
$$

(iii) On taking the values of $\delta=\gamma=1$ in Eq. (2.1), we find a new particular case of the extended generalized zeta function $\Phi_{\delta}^{*}(\xi, s, v)$ established by Goyal and Laddha [9]:

$$
\begin{aligned}
& \Phi_{\varsigma}^{*}(\xi, s, v ; p, q)=\Phi_{1, \varsigma, 1}(\xi, s, v ; p, q)=\sum_{m=0}^{\infty} \frac{B_{p, q}(\varsigma+m, 1-\varsigma)}{B(\varsigma, 1-\varsigma)} \frac{\xi^{m}}{(m+v)^{s}} \\
& (\Re(p)>0, \Re(q)>0 ; \varsigma \in \mathbb{C} ; v \neq\{0,-1,-2, \ldots\} ; s \in \mathbb{C} \text { when }|\xi|<1 \\
& \Re(s+\delta-1)>1 \text { when }|\xi|=1) .
\end{aligned}
$$

(iv) In Eq. (2.4) if we set $p=q=1$ and $\delta=\gamma=1$ gives the special case of the Hurwitz-Lerch zeta function introduced in [8, p. 160, Eq. (2.3)]:

$$
\begin{aligned}
& \Phi_{\varsigma}^{*}(\xi, s, v ; 1,1)=\Phi_{1, \varsigma, 1}(\xi, s, v ; 1,1)=\sum_{m=0}^{\infty} \frac{B_{p, q}(\varsigma+m, 1-\varsigma)}{B(\varsigma, 1-\varsigma)} \frac{\xi^{m}}{(m+v)^{s}} \\
& (\varsigma \in \mathbb{C} ; v \neq\{0,-1,-2, \ldots\} ; s \in \mathbb{C} \text { when }|\xi|<1 ; \\
& \Re(s+\delta-1)>1 \text { when }|\xi|=1) .
\end{aligned}
$$

(v) The limiting case of new extension of the generalized Hurwitz-Lerch zeta function involving extended beta function $\Phi_{\zeta ; \gamma}^{*}(\xi, s, v ; p, q)$ is given by

$$
\begin{aligned}
& \Phi_{\varsigma ; \gamma}^{*}(\xi, s, v ; p, q)=\lim _{|\delta| \rightarrow \infty}\left\{\Phi_{\varsigma ; \gamma}^{*}\left(\frac{\xi}{\delta}, s, v ; p, q\right)\right\} \\
&=\sum_{m=0}^{\infty} \frac{B_{p, q}(\varsigma+m, \gamma-\varsigma)}{B(\varsigma, \gamma-\varsigma) m !} \frac{\xi^{m}}{(m+v)^{s}} \\
&(p \geq 0, q \geq 0 ; \varsigma \in \mathbb{C} ; \gamma, v \neq\{0,-1,-2, \ldots\} ; s \in \mathbb{C} \text { when }|\xi|<1 ; \\
&\Re(s+\gamma-\varsigma)>1 \text { when }|\xi|=1) .
\end{aligned}
$$


(vi) The particular cases of Eqs. (2.1) and (2.4) are clearly seen to reduce to Eqs. (1.4) and (1.2), respectively, provided $p=q=0$, in view of the underlying connection of the functions:

$$
\Phi_{\delta, \zeta ; \gamma}(\xi, s, v ; 0,0)=\Phi_{\delta, \varsigma ; \gamma}(\xi, s, v) \quad \text { and } \quad \Phi_{1, \zeta ; 1}(\xi, s, v ; 0,0)=\Phi_{\varsigma}^{*}(\xi, s, v)
$$

Moreover, if $p=q=0$ in Eq. (2.2) yields the underlying particular case of the generalized Hurwitz-Lerch zeta function of Lin and Srivastava [13] with $(\eta=\omega=1)$ :

$$
\Phi_{1, \zeta ; \zeta}(\xi, s, v ; 0,0)=\Phi_{\varsigma ; \gamma}^{1,1}(\xi, s, v)
$$

\section{Integral representations differential formula}

The section deals with the integral representation of the new extension of the generalized Hurwitz-Lerch zeta function involving the extended beta function (2.1) as follows.

Theorem 3.1 For $\mathfrak{N}(p) \geqq 0, \mathfrak{R}(q) \geqq 0 ; p=0, \mathfrak{R}(v)>0$; $\mathfrak{N}(s)>0$, when $|\xi| \leqq 1 ; \mathfrak{R}(s)>1$, when $\xi=1$, then

$$
\Phi_{\delta, 5 ; \gamma}(\xi, s, v ; p, q)=\frac{1}{\Gamma(s)} \int_{0}^{\infty} t^{s-1} e^{-v t} F_{p, q}\left(\delta, \varsigma ; \gamma ; \xi e^{-v t}\right) d t
$$

Proof We know that the Eulerian integral of the gamma function obeys the following identity [18]:

$$
\frac{1}{(m+v)^{s}}=\frac{1}{\Gamma(s)} \int_{0}^{\infty} t^{s-1} e^{-(m+v) t} d t \quad\left(\min \{\Re(s), \Re(v)\}>0 ; m \in \mathbb{N}_{0}\right) .
$$

Employing the above result in Eq. (2.1) and then interchanging the order of summation and integration (condition above), we obtain

$$
\Phi_{\delta, \varsigma ; \gamma}(\xi, s, v ; p, q)=\frac{1}{\Gamma(s)} \int_{0}^{\infty} t^{s-1} e^{-v t}\left(\sum_{m=0}^{\infty}(\delta)_{m} \frac{B_{p, q}(\varsigma+m, \gamma-\varsigma)}{B(\varsigma, \gamma-\varsigma)} \frac{\left(\xi e^{-t}\right)^{m}}{m !}\right) d t
$$

In view of the definition (1.12) and (1.13), we obtain the required result (3.1).

Theorem 3.2 For $\mathfrak{R}(p)>0, \mathfrak{R}(q)>0 ; p=0, q=0, \mathfrak{R}(\varsigma)>0, \mathfrak{R}(\gamma)>0, \min \{s, v\}>0$, then

$$
\begin{aligned}
\Phi_{\delta, \zeta ; \gamma}(\xi, s, v ; p, q)= & \frac{e^{-p-q} \Gamma(\gamma)}{\Gamma(\varsigma) \Gamma(\gamma-\varsigma)} \\
& \times \int_{0}^{\infty} \frac{x^{\varsigma^{-1}}}{(1+x)^{\gamma}} \exp \left(-q x-\frac{p}{x}\right) \Phi_{\delta}^{*}\left(\frac{\xi x}{1+x}, s, v\right) d x
\end{aligned}
$$

and

$$
\begin{aligned}
\Phi_{\delta, \varsigma ; \gamma}(\xi, s, v ; p, q)= & \frac{e^{-p-q} \Gamma(\gamma)}{\Gamma(\varsigma) \Gamma(s) \Gamma(\gamma-\varsigma)} \int_{0}^{\infty} \int_{0}^{\infty} \frac{t^{s-1} e^{-v t} x^{\varsigma-1}}{(1+x)^{\gamma-1}} \\
& \times \exp \left(-q x-\frac{p}{x}\right)\left(1-\frac{\xi x e^{-t}}{1+x}\right)^{-\delta}
\end{aligned}
$$

provided the integrals in the right-hand side of Eqs. (3.2) and (3.3) converge. 
Proof On setting $\delta_{1}=\varsigma+m$ and $\delta_{2}=\gamma-\varsigma$ in the underlying integral representation of the extended beta function (see, e.g., [1, p. 361, Eq. (2.6)]):

$$
B_{p, q}\left(\delta_{1}, \delta_{2}\right)=e^{-p-q} \int_{0}^{\infty} \frac{x^{\delta_{1}-1}}{(1+x)^{\delta_{1}+\delta_{2}}} \exp \left(-q x-\frac{p}{x}\right) d x
$$

we obtain

$$
B_{p, q}(\varsigma+m, \gamma-\varsigma)=e^{-p-q} \int_{0}^{\infty} \frac{x^{\varsigma+m-1}}{(1+x)^{\gamma+m}} \exp \left(-q x-\frac{p}{x}\right) d x
$$

in view of the above relation and using definition (1.2), Eq. (2.1) clearly gives the first statement of Theorem 3.2.

Moreover, using the integral representation (1.5) in Eq. (3.4), we obtain the required result (3.3).

Theorem 3.3 For $\Re(p)>0, \mathfrak{i}(q)>0 ; p=0, q=0, \mathfrak{R}(\varsigma)>0, \mathfrak{R}(\gamma)>0$, $\min \{s, v\}>0$, then

$$
\begin{aligned}
\Phi_{\delta, \varsigma ; \gamma}(\xi, s, v ; p, q)= & \frac{2^{1-\gamma} \Gamma(\gamma)}{\Gamma(\varsigma) \Gamma(\gamma-\varsigma)} \int_{-1}^{1}(1+x)^{\varsigma-1}(1-x)^{\gamma-\varsigma-1} \\
& \times \exp \left(-\frac{2(p+q)+2(q-p)}{1-x^{2}}\right) \Phi_{\delta}^{*}\left(\frac{\xi(1+x)}{2}, s, v\right) d x
\end{aligned}
$$

and

$$
\begin{aligned}
\Phi_{\delta, \zeta ; \gamma}(\xi, s, v ; p, q)= & \frac{2^{1-\gamma} \Gamma(\gamma)}{\Gamma(\varsigma) \Gamma(s) \Gamma(\gamma-\varsigma)} \int_{-1}^{1} \int_{0}^{\infty} t^{s-1} e^{-v t}(1+x)^{\varsigma-1}(1-x)^{\gamma-\varsigma-1} \\
& \times \exp \left(-\frac{2(p+q)+2(q-p)}{1-x^{2}}\right)\left(1-\frac{\xi e^{-t}(1+x)}{2}\right)^{-\delta} d x .
\end{aligned}
$$

Proof On setting $\delta_{1}=\varsigma+m$ and $\delta_{2}=\gamma-\varsigma$ in the underlying integral representation of the extended beta function (see, e.g., [1, p. 361, Eq. (2.7)]):

$$
B_{p, q}\left(\delta_{1}, \delta_{2}\right)=2^{1-\delta_{1}-\delta_{2}} \int_{-1}^{\infty}(1+x)^{\delta_{1}-1}(1-x)^{\delta_{2}-1} \exp \left(-\frac{2(p+q)+2(q-p)}{1-x^{2}}\right) d x
$$

similarly, it can be easy to prove both the assertions of Theorem 3.3 and of Theorem 3.2.

Theorem 3.4 For $\Re(p)>0, \Re(q)>0 ; p=0, q=0, \mathfrak{R}(\varsigma)>0, \mathfrak{R}(\gamma)>0, \min \{s, v\}>0$, then

$$
\begin{aligned}
\Phi_{\delta, \varsigma ; \gamma}(\xi, s, v ; p, q)= & \frac{(c-a) \Gamma(\gamma)}{\Gamma(\varsigma) \Gamma(\gamma-\varsigma)} \int_{a}^{c}(x-a)^{\varsigma-1}(c-x)^{\gamma-\varsigma-1} \\
& \times \exp \left(-\frac{(c-a)}{(x-a)(c-x)}\{(q-p) x+(p c-q a)\}\right) \\
& \times \Phi_{\delta}^{*}\left(\xi\left(\frac{x-a}{c-a}\right), s, v\right) d x
\end{aligned}
$$


and

$$
\begin{aligned}
\Phi_{\delta, \zeta ; \gamma}(\xi, s, v ; p, q)= & \frac{(c-a) \Gamma(\gamma)}{\Gamma(\varsigma) \Gamma(s) \Gamma(\gamma-\varsigma)} \int_{a}^{c}(x-a)^{\varsigma-1}(c-x)^{\gamma-\varsigma-1} \\
& \times \exp \left(-\frac{(c-a)}{(x-a)(c-x)}\{(q-p) x+(p c-q a)\}\right) \\
& \times\left(1-\xi\left(\frac{x-a}{c-a}\right) e^{-t}\right)^{-\delta} d x .
\end{aligned}
$$

Proof On setting $\delta_{1}=\varsigma+m$ and $\delta_{2}=\gamma-\varsigma$ in the underlying integral representation of the extended beta function (see, e.g., [1, p. 362, Eq. (2.8)]):

$$
\begin{aligned}
B_{p, q}\left(\delta_{1}, \delta_{2}\right)= & (c-a)^{1-\delta_{1}-\delta_{2}} \int_{a}^{c}(x-a)^{\delta_{1}-1}(c-x)^{\delta_{2}-1} \\
& \times \exp \left(-\frac{(c-a)}{(x-a)(c-x)}\{(q-p) x+(p c-q a)\}\right) d x,
\end{aligned}
$$

similarly, it is easy to prove both the assertions of Theorem 3.4 and of Theorem 3.2.

Theorem 3.5 For $p \geq 0, q \geq 0, \Re(\delta)>0, \Re(v)>0, \Re(s)>0$, when $|\xi| \leqq 1(\xi \neq 1) ; \Re(s)>1$, when $\xi=1$, then

$$
\Phi_{\delta, \zeta, \gamma ; p, q}(\xi, s ; v ; p, q)=\frac{1}{\Gamma(\delta)} \int_{0}^{\infty} t^{\delta-1} e^{-t} \Phi_{\zeta, \gamma ; p, q}^{*}(\xi t, s ; v ; p, q) d t
$$

where $\Phi_{5, \gamma ; p, q}^{*}(\xi t, s ; v ; p, q)$ is the limiting case in (2.6).

Proof The integral representation of the Pochhammer symbol $(\delta)_{m}$ is defined as

$$
(\delta)_{m}=\frac{1}{\Gamma(\delta)} \int_{0}^{\infty} t^{\delta+m-1} e^{-t} d t
$$

By making use of the above relation in (2.1) and interchanging the order of summation and integration which may be admissible subject to the condition of Theorem 3.5, we obtain

$$
\Phi_{\delta, \zeta, \gamma ; p, q}(\xi, s ; v ; p, q)=\frac{1}{\Gamma(\delta)} \int_{0}^{\infty} t^{\delta-1} e^{-t} \sum_{m=0}^{\infty} \frac{B_{p, q}(\varsigma+m, \gamma-\varsigma)}{B(\varsigma, \gamma-\varsigma)} \frac{(\xi t)^{m}}{m !(m+v)^{s}} d t
$$

Applying (2.6), we get the required integral representation.

Remark 3.1 On substituting $q=0$ in Eq. (3.13), we obtain the result earlier obtained by Parmar and Raina [8].

Subsequently, we establish the underlying derivative formula of (2.1).

Theorem 3.6 The following differential formula for $\Phi_{\delta, 5, \gamma ; p, q}(\xi, s ; v ; p, q)$ holds:

$$
\frac{d^{m}}{d \xi^{m}}\left\{\Phi_{\delta, \zeta, \gamma ; p, q}(\xi, s ; v ; p, q)\right\}=\frac{(\delta)_{m}(\varsigma)_{m}}{(\gamma)_{m}} \Phi_{\delta+m, \varsigma+m, \gamma+m ; p, q}(\xi, s ; v+m ; p, q) \quad(m \in \mathbb{N})
$$


Proof Consider the derivative of (2.1) with respect to $\xi$, we obtain

$$
\frac{d}{d \xi}\left\{\Phi_{\delta, \varsigma, \gamma ; p, q}(\xi, s ; v ; p, q)\right\}=\sum_{m=0}^{\infty} \frac{(\delta)_{m}}{(m-1) !} \frac{B_{p, q}(\varsigma+m, \gamma-\varsigma)}{B(\varsigma, \gamma-\varsigma)} \frac{\xi^{m-1}}{(m+v)^{s}}
$$

which upon replacing $m$ by $m+1$ in Eq. (3.14) and employing the identity

$$
B(\varsigma, \gamma-\varsigma)=\frac{\gamma}{\varsigma} B(\varsigma+1, \gamma-\varsigma), \quad(v)_{m+1}=v(v+1)_{m},
$$

leads to the derivative formula

$$
\frac{d}{d \xi}\left\{\Phi_{\delta, \varsigma, \gamma ; p, q}(\xi, s ; v ; p, q)\right\}=\frac{\delta \varsigma}{\gamma} \Phi_{\delta+1, \varsigma+1, \gamma+1 ; p, q}(\xi, s ; v+1 ; p, q),
$$

recursive application of this procedure provides the required result (3.6).

\section{Mellin transform of the Hurwitz-Lerch zeta function and their relation between $\bar{H}$-function}

The Mellin transform of a suitable integrable function $f(\kappa)$ with index $\varphi$ is defined, generally, by

$$
\mathcal{M}\{f(\kappa): \kappa \rightarrow \varphi\}=\int_{0}^{\infty} \kappa^{\varphi-1} f(\kappa) d \kappa \quad(0<\kappa<\infty)
$$

Theorem 4.1 Consider $\mathfrak{R}(p)>0, \mathfrak{R}(q)>0, \mathfrak{R}(\varphi)>0, \mathfrak{R}(\psi)>0, \mathfrak{R}(\varsigma+m+\varphi)>0, \mathfrak{R}(\gamma-$ $\varsigma+\psi)>0$, then the Mellin transform of the function $\Phi_{\delta, 5, \gamma ; p, q}(\xi, s ; v ; p, q)$ defined by $(2.1)$ is given by

$$
\begin{aligned}
\mathcal{M}\left\{\Phi_{\delta, \varsigma, \gamma ; p, q}(\xi, s ; v ; p, q): p \rightarrow \varphi, q \rightarrow \psi\right\} \\
=\frac{\Gamma(\varphi) \Gamma(\psi) B(\varsigma+\varphi, \gamma-\varsigma+\psi)}{B(\varsigma, \gamma-\varsigma)} \Phi_{\delta, \varsigma+\varphi ; \varphi+\psi+\gamma}(\xi, s ; v) .
\end{aligned}
$$

Proof Consider the Mellin transform for (2.1) in view of definition (4.1), we obtain

$$
\begin{aligned}
\mathcal{M} & \left\{\Phi_{\delta, \varsigma, \gamma ; p, q}(\xi, s ; v ; p, q): p \rightarrow \varphi, q \rightarrow \psi\right\} \\
& =\int_{0}^{\infty} \int_{0}^{\infty} p^{\varphi-1} q^{\psi-1} \Phi_{\delta, \varsigma, \gamma ; p, q}(\xi, s ; v ; p, q) d p d q \\
& =\int_{0}^{\infty} \int_{0}^{\infty} p^{\varphi-1} q^{\psi-1} \sum_{m=0}^{\infty} \frac{B_{p, q}(\varsigma+m, \gamma-\varsigma)}{B(\varsigma, \gamma-\varsigma)} \frac{(\delta)_{m} \xi^{m}}{m !(m+v)^{s}} d p d q \\
& =\sum_{m=0}^{\infty} \frac{(\delta)_{m} \xi^{m}}{m !(m+v)^{s}} \frac{1}{B(\varsigma, \gamma-\varsigma)} \int_{0}^{\infty} \int_{0}^{\infty} p^{\varphi-1} q^{\psi-1} B_{p, q}(\varsigma+m, \gamma-\varsigma) d p d q .
\end{aligned}
$$

Employing the underlying well-known integral representation (see [1, p. 360, Eq. (1.14)])

$$
\begin{aligned}
& \int_{0}^{\infty} \int_{0}^{\infty} p^{\varphi-1} q^{\psi-1} B_{p, q}(\varsigma+m, \gamma-\varsigma) d p d q=\Gamma(\varphi) \Gamma(\psi) B(\varsigma+m+\varphi, \gamma-\varsigma+\psi) \\
& (\Re(\varphi)>0, \Re(\psi)>0),
\end{aligned}
$$


we get

$$
\begin{aligned}
\mathcal{M} & \left\{\Phi_{\delta, \varsigma, \gamma ; p, q}(\xi, s ; v ; p, q): p \rightarrow \varphi, q \rightarrow \psi\right\} \\
= & \frac{\Gamma(\varphi) \Gamma(\psi)}{B(\varsigma, \gamma-\varsigma)} \sum_{m=0}^{\infty} \frac{(\delta)_{m}}{m !} \frac{\xi^{m}}{(m+v)^{s}} B(\varsigma+m+\varphi, \gamma-\varsigma+\psi) \\
= & \frac{\Gamma(\varphi) \Gamma(\psi) B(\varsigma+\varphi, \gamma-\varsigma+\psi)}{B(\varsigma, \gamma-\varsigma)} \sum_{m=0}^{\infty} \frac{(\delta)_{m}(\varsigma+\varphi)_{m}}{m !(\varphi+\gamma+\psi)_{m}} \frac{\xi^{m}}{(m+v)^{s}},
\end{aligned}
$$

which gives the desired result 4.1.

Remark 4.1 The generalized Hurwitz-Lerch zeta function can be easily written in terms of $\bar{H}$-function as appears in the literature $[19,20]$ (see also [7, p. 316, Eq. (3.2)]):

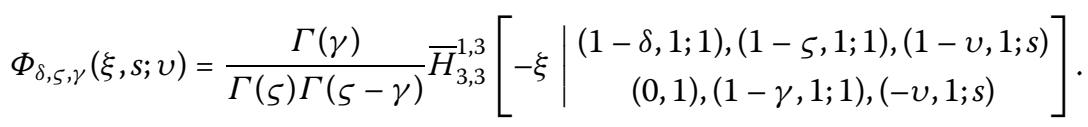

By using (4.5) we can easily deduce Eq. (4.2), Mellin representation in terms of the $\bar{H}$ function which is a fascinating result given as a corollary.

Corollary 4.1 The underlying Mellin representation holds:

$$
\begin{aligned}
& \mathcal{M}\left\{\Phi_{\delta, \zeta, \gamma}(\xi, s ; v ; p, q): p \rightarrow \varphi, q \rightarrow \psi\right\} \\
& =\frac{\Gamma(\varphi) \Gamma(\psi) \Gamma(\gamma-\varsigma+\psi)}{\Gamma(\delta) B(\varsigma, \gamma-\varsigma)} \bar{H}_{3,3}^{1,3}\left[\begin{array}{r|c}
(1-\delta, 1 ; 1),(1-\varsigma-\varphi, 1 ; 1),(1-v, 1 ; s) \\
(0,1),(1-\varphi-\psi-\gamma, 1 ; 1),(-v, 1 ; s)
\end{array}\right] .
\end{aligned}
$$

Theorem 4.2 For $p \geqq 0, q \geqq 0, \delta \in \mathbb{C}$ and $|t|<1$, the underlying generating function holds:

$$
\sum_{m=0}^{\infty}(\delta)_{m} \Phi_{\delta+m, 5, \gamma}(\xi, s ; v ; p, q) \frac{t^{m}}{m !}=(1-t)^{-\delta} \Phi_{\delta+m, s, \gamma}\left(\frac{\xi}{1-t}, s, v ; p, q\right) .
$$

Proof Consider the left-hand side of the assertion (4.7) of Theorem 4.2 be denoted by $K_{1}$ and in view of definition (2.1), we obtain

$$
K_{1}=\sum_{m=0}^{\infty}(\delta)_{m}\left\{\sum_{l=0}^{\infty}(\delta+m)_{l} \frac{B_{p, q}(\varsigma+l, \gamma-\varsigma)}{B(\varsigma, \gamma-\varsigma)} \frac{\xi^{l}}{l !(m+v)^{s}}\right\} \frac{t^{m}}{m !}
$$

Inverting the order of summation of the above equality and after a little simplification, we get

$$
=\sum_{l=0}^{\infty}(\delta)_{l} \frac{B_{p, q}(\varsigma+l, \gamma-\varsigma)}{B(\varsigma, \gamma-\varsigma)}\left\{\sum_{m=0}^{\infty}(\delta+l)_{m} \frac{t^{m}}{m !}\right\} \frac{\xi^{l}}{l !(m+v)^{s}} .
$$

Now, employing the binomial expansion

$$
(1-t)^{-(\delta+l)}=\sum_{m=0}^{\infty}(\delta+l)_{m} \frac{t^{m}}{m !}
$$

and in view of definition (2.1), we obtain the assertion (4.7) of Theorem 4.2. 
Theorem 4.3 Let $p, q \geqq 0, \delta \in \mathbb{C}$ and $|t|<|v| ; s \neq 1$ then the generating functions of $\Phi_{\delta, 5, \gamma}(\xi, s ; v ; p, q)$ is given by

$$
\sum_{m=0}^{\infty} \frac{(s)_{m}}{m !} \Phi_{\delta, 5, \gamma}(\xi, s+m ; v ; p, q) t^{m}=\Phi_{\delta, 5, \gamma}(\xi, s ; v-t ; p, q) .
$$

More generally

$$
\begin{aligned}
& \sum_{m=0}^{\infty} \frac{(\delta)_{m}}{m !} \Phi_{\delta, 5, \gamma}(\xi, s+m ; v ; p, q) t^{m} \\
& \quad=\sum_{l=0}^{\infty} \frac{(\delta)_{l}}{l !} \frac{B_{p, q}(\varsigma+l, \gamma-\varsigma)}{B(\varsigma, \gamma-\varsigma)} \frac{\xi^{l}}{(l+v)^{s-\delta}(l+v-t)^{\delta}}
\end{aligned}
$$

and

$$
\begin{aligned}
& \sum_{m=0}^{\infty} \Phi_{\delta, \zeta, \gamma}(\xi, s+m ; v ; p, q) \frac{t^{m}}{m !} \\
& \quad=\sum_{l=0}^{\infty} \frac{(\delta)_{l}}{l !} \frac{B_{p, q}(\varsigma+l, \gamma-\varsigma)}{B(\varsigma, \gamma-\varsigma)} \frac{\xi^{l}}{(l+v)^{s}} \exp \left(\frac{t}{l+v}\right) .
\end{aligned}
$$

Proof Using the definition (2.1) in the right-hand side of (4.10), we have

$$
\begin{aligned}
\Phi_{\delta, \varsigma, \gamma}(\xi, s ; v-t ; p, q) & =\sum_{l=0}^{\infty}(\delta)_{l} \frac{B_{p, q}(\varsigma+l, \gamma-\varsigma)}{B(\varsigma, \gamma-\varsigma)} \frac{\xi^{l}}{l !(l+v-t)^{s}} \\
& =\sum_{l=0}^{\infty}(\delta)_{l} \frac{B_{p, q}(\varsigma+l, \gamma-\varsigma)}{B(\varsigma, \gamma-\varsigma)} \frac{\xi^{l}}{l !(l+v)^{s}}\left(1-\frac{t}{l+v}\right)^{-s} .
\end{aligned}
$$

In view of expansion (4.9) and some little simplification of the above second equality, we are thus led to the assertion (4.11).

The generating function (4.11) can easily be deduced by substituting $\delta=s$ in Eq. (4.12).

\section{Fractional kinetic equation}

This section deals with the fractional kinetic equation (FKE) involving the new extended Hurwitz-Lerch zeta function (2.1). The FKE has great significance in the field of astrophysics and mathematical physics.

The solutions of FKE has many applications in various fields such as renormalization of the non-stationary problem near the phase transition point [21], the theory of turbulence [22], diffusion in porous media [23], and kinetics in viscoelastic media [24], which has been published in the literature of special functions.

In 2000 Haubold and Mathai [25] derived a fascinating result between the rate of change of reaction, the destruction rate, and the production rate given by

$$
\frac{d \mathcal{N}}{d t}=-\delta\left(\mathcal{N}_{t}\right)+\mathfrak{p}\left(\mathcal{N}_{t}\right)
$$

where $\mathcal{N}=\mathcal{N}(t)$ is the rate of reaction, $\delta\left(\mathcal{N}_{t}\right)=: \delta$ is the rate of destruction, $\mathfrak{p}=\mathfrak{p}(\mathcal{N})$ is the rate of production and $\mathcal{N}_{t}$ signifies the function defined by $\mathcal{N}_{t}\left(t^{*}\right)=\mathcal{N}\left(t-t^{*}\right) ; t^{*}>0$. 
Under spatial fluctuations or homogeneities where the quantity $\mathcal{N}(t)$ is neglected we arrive at a particular case of Eq. (5.1), which is given by (see $[25,26])$

$$
\frac{d \mathcal{N}_{i}}{d t}=-c_{i} \mathcal{N}_{i}(t)
$$

with the initial condition $\mathcal{N}_{i}(t=0)=\mathcal{N}_{0}$ standing for the number of density of species $i$ at time $t=0 ; c_{i}>0$, studied as standard kinetic equation. Integrating both sides of Eq. (5.2) under the condition if the index $i$ is omitted, we get

$$
\mathcal{N}(t)-\mathcal{N}_{0}=-c_{0}{ }_{0} D_{t}^{-1}-\mathcal{N}(t)
$$

where ${ }_{0} D_{t}^{-1}$ denotes the standard fractional integral operator.

A fractional generalization of standard kinetic Eq. (5.2) is investigated by Haubold and Mathai [25] as follows:

$$
\mathcal{N}(t)-\mathcal{N}_{0}=-c_{0}^{\omega} D_{t}^{-\omega}-\mathcal{N}(t)
$$

where ${ }_{0} D_{t}^{-\omega}$ is the familiar Riemann-Liouville fractional integral operator (see [27]) defined as

$$
{ }_{0} D_{t}^{-\omega} f(t)=\frac{1}{\Gamma(\omega)} \int_{0}^{t}(t-u)^{\omega-1} f(u) d u \quad(t>0, \Re(\omega)>0),
$$

and they obtained the solution of (5.5) as follows:

$$
\mathcal{N}(t)=\mathcal{N}_{0} \sum_{l=0}^{\infty} \frac{(-1)^{l}}{\Gamma(\omega l+1)}(c t)^{\omega k}
$$

Moreover, Saxena and Kalla [25] obtained the underlying fractional kinetic equation:

$$
\mathcal{N}(t)-\mathcal{N}_{0} f(t)=-c_{0}^{\omega}{ }_{0} D_{t}^{-\omega} \mathcal{N}(t) \quad(\Re(\omega)>0)
$$

where $\mathcal{N}(t)$ is the number density of a given species at time $t, \mathcal{N}_{0}=\mathcal{N}(0)$ is the number density of that species at time $t=0, c$ is a constant and $f \in \mathcal{L}(0, \infty)$.

Applying the Laplace transform to (5.7) (see [28]),

$$
\begin{gathered}
L\{\mathcal{N}(t) ; \mathfrak{p}\}=\mathcal{N}_{0}\left(\sum_{m=0}^{\infty}\left(-c^{\omega}\right)^{m} \mathfrak{p}^{-m \omega}\right) F(\mathfrak{p}) \\
\left(m \in \mathbb{N}_{0},\left|\frac{c}{\mathfrak{p}}\right|<1\right), \\
F(\mathfrak{p})=L\{f(t) ; \mathfrak{p}\}=\int_{0}^{\infty} e^{-\mathfrak{p} t} f(t) d t \quad(\Re(\mathfrak{p})>0) .
\end{gathered}
$$

Now, we proceed to obtain the solution of the generalized fractional; kinetic equations by considering our new extended Hurwitz-Lerch zeta function. The result obtained in terms of the generalized Mittag-Leffler function. 
Theorem 5.1 Let $d>0, \omega>0$ and $v \neq d, c>0$ then the solution of the equation

$$
\mathcal{N}(t)-\mathcal{N}_{0}\left\{\Phi_{\delta, \zeta ; \gamma}\left(t^{\omega} \xi, s, v ; p, q\right)\right\}=-d_{0}^{\omega}{ }_{0} D_{t}^{-\omega} \mathcal{N}(t)
$$

is given by the following relation:

$$
\begin{aligned}
\mathcal{N}(t)= & \mathcal{N}_{0} \sum_{m=0}^{\infty} \frac{B_{p, q}(\varsigma+m, \gamma-\varsigma)}{B(\varsigma, \gamma-\varsigma)} \frac{(\delta)_{m}}{m !} \frac{\Gamma(m \omega+m+1) t^{m+m \omega}}{(m+v)^{s}} \\
& \times E_{\omega, m+m \omega+1}\left(d^{\omega} t^{\omega}\right)
\end{aligned}
$$

where the $E_{\alpha, \beta}(\xi)$ denotes the generalized Mittag-Leffler function [29] is given by

$$
E_{\alpha, \beta}(\xi)=\sum_{m=0}^{\infty} \frac{\xi^{m}}{\Gamma(\alpha m+\beta)} \quad(\Re(\alpha)>0, \mathfrak{R}(\beta)>0) .
$$

Proof The Laplace transform of the Riemann-Liouville fractional integral operator is given by [30]

$$
L\left\{{ }_{0} D_{t}^{-\omega} f(t) ; \mathfrak{p}\right\}=\mathfrak{p}^{-\omega} F(\mathfrak{p})
$$

where $F(\mathfrak{p})$ is defined in (5.9).

Now, taking the Laplace transform of both sides of Eq. (5.11), we obtain

$$
\begin{aligned}
& L\{\mathcal{N}(t) ; \mathfrak{p}\}=\mathcal{N}_{0} L\left\{\Phi_{\delta, \varsigma ; \gamma}\left(t^{\omega} \xi, s, v ; p, q\right) ; \mathfrak{p}\right\}-d_{0}^{\omega}\left\{{ }_{0} D_{t}^{-\omega} \mathcal{N}(t) ; \mathfrak{p}\right\} \\
& \mathcal{N}(\mathfrak{p})=\mathcal{N}_{0}\left(\int_{0}^{\infty} e^{-\mathfrak{p} t} \sum_{m=0}^{\infty} \frac{B_{p, q}(\varsigma+m, \gamma-\varsigma)}{B(\varsigma, \gamma-\varsigma)} \frac{(\delta)_{m}}{m !} \frac{\left(t^{\omega+1}\right)^{m}}{(m+v)^{s}} d t\right)-d^{\omega} \mathfrak{p}^{-\omega} \mathcal{N}(\mathfrak{p}) \\
& \mathcal{N}(\mathfrak{p})+d^{\omega} \mathfrak{p}^{-\omega} \mathcal{N}(\mathfrak{p})=\mathcal{N}_{0} \sum_{m=0}^{\infty} \frac{B_{p, q}(\varsigma+m, \gamma-\varsigma)}{B(\varsigma, \gamma-\varsigma)} \frac{(\delta)_{m}}{m !} \frac{1}{(m+v)^{s}} \int_{0}^{\infty} e^{-\mathfrak{p} t} t^{\omega m+m} d t \\
& \mathcal{N}(\mathfrak{p})+d^{\omega} \mathfrak{p}^{-\omega} \mathcal{N}(\mathfrak{p})=\mathcal{N}_{0} \sum_{m=0}^{\infty} \frac{B_{p, q}(\varsigma+m, \gamma-\varsigma)}{B(\varsigma, \gamma-\varsigma)} \frac{(\delta)_{m}}{m !} \frac{1}{(m+v)^{s}} \frac{\Gamma(m \omega+m+1)}{\mathfrak{p}^{m \omega+m+1}} \\
& \mathcal{N}(\mathfrak{p})= \\
& \quad \mathcal{N}_{0} \sum_{m=0}^{\infty} \frac{B_{p, q}(\varsigma+m, \gamma-\varsigma)}{B(\varsigma, \gamma-\varsigma)} \frac{(\delta)_{m}}{m !} \frac{1}{(m+v)^{s}} \\
& \quad \times \Gamma(m \omega+m+1) \mathfrak{p}^{-(m \omega+m+1)}\left(1+d^{\omega} \mathfrak{p}^{-\omega}\right)^{-1} \\
& \mathcal{N}(\mathfrak{p})= \\
& \quad \mathcal{N}_{0} \sum_{m=0}^{\infty} \frac{B_{p, q}(\varsigma+m, \gamma-\varsigma)}{B(\varsigma, \gamma-\varsigma)} \frac{(\delta)_{m}}{m !} \frac{1}{(m+v)^{s}} \\
& \quad \times \Gamma(m \omega+m+1) \mathfrak{p}^{-(m \omega+m+1)} \sum_{r=0}^{\infty}\left(d^{\omega} \mathfrak{p}^{-\omega}\right)^{r} .
\end{aligned}
$$


Applying the inverse Laplace transform to (5.13), we obtain

$$
\begin{aligned}
L^{-1}\{\mathcal{N}(\mathfrak{p})\}= & \mathcal{N}_{0} \sum_{m=0}^{\infty} \frac{B_{p, q}(\varsigma+m, \gamma-\varsigma)}{B(\varsigma, \gamma-\varsigma)} \frac{(\delta)_{m}}{m !} \frac{1}{(m+v)^{s}} \\
& \times \Gamma(m \omega+m+1) L^{-1}\left\{\sum_{r=0}^{\infty} d^{\omega r} \mathfrak{p}^{-\omega r-m \omega-m-1}\right\}, \\
\mathcal{N}(t)= & \mathcal{N}_{0} \sum_{m=0}^{\infty} \frac{B_{p, q}(\varsigma+m, \gamma-\varsigma)}{B(\varsigma, \gamma-\varsigma)} \frac{(\delta)_{m}}{m !} \frac{1}{(m+v)^{s}} \\
& \times \Gamma(m \omega+m+1)\left\{\sum_{r=0}^{\infty} d^{\omega r} \frac{t^{\omega r+m \omega+m 1}}{\Gamma(\omega r+m \omega+m+1)}\right\}, \\
\mathcal{N}(t)= & \mathcal{N}_{0} \sum_{m=0}^{\infty} \frac{B_{p, q}(\varsigma+m, \gamma-\varsigma)}{B(\varsigma, \gamma-\varsigma)} \frac{(\delta)_{m}}{m !} \frac{1}{(m+v)^{s}} \\
& \times \Gamma(m \omega+m+1) t^{m \omega+m}\left\{\sum_{r=0}^{\infty} \frac{d^{\omega r} t^{\omega r}}{\Gamma(\omega r+m \omega+m+1)}\right\}, \\
& \times \Gamma(m \omega+m+1) t^{m \omega+m} E_{\omega, m \omega+m+1}\left(d^{\omega} t^{\omega}\right), \\
\mathcal{N}(t)= & \mathcal{N}_{0} \sum_{m=0}^{\infty} \frac{B_{p, q}(\varsigma+m, \gamma-\varsigma)}{B(\varsigma, \gamma-\varsigma)} \frac{(\delta)_{m}}{m !} \frac{1}{(m+v)^{s}} \\
& \Gamma(m)
\end{aligned}
$$

which is the required result.

\section{Concluding remarks}

In the present paper, it seems to be of interest that the extensions of Hurwitz-Lerch zeta function so obtained are very general in nature and, by specific parameters, can yield the previously defined Hurwitz-Lerch zeta function which is shown in this paper. On that account, they become of great importance from an application perspective. For example, here we establish the connection of the new extended Hurwitz-Lerch zeta function with other special functions.

By using the connection of the generalization of the extended beta function with other special functions (see [1, p. 367, Sect. 5]), we can obtain the relation between the new extended Hurwitz-Lerch zeta function with other special functions.

- Laguerre polynomials

$$
\begin{aligned}
\Phi_{\delta, \zeta ; \gamma}(\xi, s, v ; p, q)= & \sum_{m=0}^{\infty} \sum_{n, r=0}^{\infty} \frac{(\delta)_{m}}{m !} \frac{B(\varsigma+m+n+1, \gamma-\varsigma+r+1)}{B(\varsigma, \gamma-\varsigma)} \\
& \times \frac{\xi^{m}}{(m+v)^{s}} L_{n}(p) L_{r}(q) \\
(\Re(\varsigma+m+n)>- & 1, \Re(\gamma-\varsigma+r)>-1, \Re(\varsigma)>0, \Re(\gamma)>\mathfrak{N}(\varsigma)) .
\end{aligned}
$$


- Meijer's G-function

$$
\begin{aligned}
& \Phi_{\delta, \varsigma ; \gamma}(\xi, s, v ; p, q) \\
& =\sum_{m=0}^{\infty} \frac{(\delta)_{m}}{m !} \frac{1}{B(\varsigma, \gamma-\varsigma)} \frac{\xi^{m}}{(m+v)^{s}} \\
& \quad \times G_{0,0: 2,2 ; 0 ; 2,0}^{1,0: 0,0 ; 2}\left[\begin{array}{l}
(\gamma+m ; 1,1):-; \\
-,(0,1),(\varsigma+m, 1) ;(0,1),(\gamma-\varsigma, 1) ;
\end{array}\right], q, q
\end{aligned}
$$

where $G$ represents Meijer's G-function (see [31, p. 7, Eq. (1.2.3) and p. 88, Eq. (6.4.1)]).

- Appell series

$$
\begin{aligned}
& \Phi_{\delta, 5 ; \gamma}(\xi, s, v ; p, q) \\
& =\sum_{m=0}^{\infty} \frac{(\delta)_{m}}{m !} \frac{1}{B(\varsigma, \gamma-\varsigma)} \frac{\xi^{m}}{(m+v)^{s}} \\
& \quad \times B(\varsigma+m, \gamma-\varsigma) F_{2}[1-m-\gamma,-,-; 1-\varsigma-m, 1-\gamma+\varsigma ;-p,-q],
\end{aligned}
$$

where $F_{2}[\cdot]$ represents one of the four Appell series $F_{j}(j=1,2,3,4)$ (see [32, pp. 22-23]).

Similarly, we can further obtain the connection with the Macdonald function and the Whittaker function.

Acknowledgements

Not applicable.

\section{Funding}

None.

Availability of data and materials

None.

Competing interests

The authors declare that they have no competing interests.

\section{Authors' contributions}

All authors contributed to writing the draft, software and reviewing the final version of the manuscript. All authors read and approved the final manuscript.

\section{Author details}

'Department of Applied Mathematics, Faculty of Engineering and Technology, Aligarh Muslim University, Aligarh, 202002, India. ${ }^{2}$ Department of Mathematics, School of Basic and Applied Sciences, Lingaya's Vidyapeeth, Faridabad, 121002, Haryana, India. ${ }^{3}$ Department of Mathematics, College of Arts and Sciences, Prince Sattam bin Abdulaziz University, Wadi Aldawaser, 11991, Saudi Arabia. ${ }^{4}$ Department of Mathematics, Cankaya University, Ankara, 06790, Turkey. ${ }^{5}$ Institute of Space Sciences, Magurele-Bucharest, 077125, Romania. ${ }^{6}$ Department of Medical Research, China Medical University Hospital, China Medical University, Taichung, 40447, Taiwan.

\section{Publisher's Note}

Springer Nature remains neutral with regard to jurisdictional claims in published maps and institutional affiliations.

Received: 23 June 2020 Accepted: 24 August 2020 Published online: 04 September 2020

\section{References}

1. Choi, J., Rathie, A.K., Parmar, R.K.: Extension of extended beta, hypergeometric and confluent hypergeometric functions. Honam Math. J. 36(2), 357-385 (2014) 
2. Erdèlyi, A., Magnus, W., Oberhettinger, F., Tricomi, F.G.: Higher Transcendental Functions, vol. I. McGraw-Hill, New York (1953)

3. Srivastava, H.M., Choi, J.: Series Associated with the Zeta and Related Functions. Kluwer Acedemic, Dordrecht (2001)

4. Srivastava, H.M.: Generating relations and other results associated with some families of the extended Hurwitz-Lerch zeta functions. SpringerPlus 2, Article ID 67 (2013)

5. Srivastava, H.M., Luo, M.J., Raina, R.K.: New results involving a class of generalized Hurwitz-Lerch zeta functions and their applications. Turk. J. Anal. Number Theory 1(1), 26-35 (2013)

6. Choi, J., Jang, D.S., Srivastava, H.M.: A generalization of the Hurwitz-Lerch zeta function. Integral Transforms Spec. Funct. 19, 65-79 (2008)

7. Garg, M., Jain, K., Kalla, S.L.: A further study of general Hurwitz-Lerch zeta function. Algebras Groups Geom. 25, 311-319 (2008)

8. Parmar, R.K., Raina, R.K.: On a certain extension of the Hurwitz-Lerch zeta function. An. Univ. Vest. Timiş., Ser. Mat.-Inform. 2, 157-170 (2014)

9. Goyal, S.P., Laddha, R.K.: On the generalized zeta function and the generalized Lambert function. Ganita Sandesh 11 99-108 (1997)

10. Nisar, K.S.: Further extension of the generalized Hurwitz-Lerch zeta function of two variables. Mathematics 7, 48 (2019)

11. Rahman, G., Nisar, K.S., Arshad, M.: A new extension of Hurwitz-Lerch Zeta function. (2018). arXiv:1802.07823 [math.CA]

12. Rahman, G., Nisar, K.S., Mubeen, S.: A (p,v)-extension of Hurwitz-Lerch Zeta function and its properties. Preprints, 2018030008 (2018). https://doi.org/10.20944/preprints201803.0008.v1

13. Lin, S.D., Srivastava, H.M.: Some families of the Hurwitz-Lerch zeta functions and associated fractional derivative and other integral representations. Appl. Math. Comput. 154, 725-733 (2004)

14. Rainville, E.D.: Special Functions. Macmillan Co., New York (1960). Reprinted by Chelsea Publ. Co., Bronx, New York (1971)

15. Raina, R.K., Chhajed, P.K.: Certain results involving a class of functions associated with the Hurwitz zeta function. Acta Math. Univ. Comen. 73, 89-100 (2004)

16. Chaudhry, M.A., Qadir, A., Raflque, M., Zubair, S.M.: Extension of Euler's beta function. J. Comput. Appl. Math. 78, 19-32 (1997)

17. Chaudhry, M.A., Qadir, A., Srivastava, H.M., Paris, R.B.: Extended hypergeometric and confluent hypergeometric functions. Appl. Math. Comput. 159, 589-602 (2004)

18. Srivastava, H.M., Choi, J.: Zeta and q-Zeta Functions and Associated Series and Integrals. Elsevier, Amsterdam (2012)

19. Inayat-Hussain, A.A.: New properties of hypergeometric series derivable from Feynman integrals. I: transformation and reduction formulae. J. Phys. A, Math. Gen. 20, 4109-4117 (1987)

20. Inayat-Hussain, A.A.: New properties of hypergeometric series derivable from Feynman integrals. II: a generalization of the H-function. J. Phys. A, Math. Gen. 20,4119-4128 (1987)

21. Hohenberg, P.C., Halperin, B.I:: Rev. Mod. Phys. 49435 (1977)

22. Novikov, E.A.: Conditionally-averaged dynamics of turbulence, new scaling and stochastic modelling. In: Shlesinger M.F., Zaslavsky, G.M., Frisch, U. (eds.) Lévy Flights and Related Topics in Physics, pp. 35-50. Springer, New York (1995)

23. Mainardi, F.: J. Alloys Compd. 211/212, 534 (1994); Chaos Solitons Fractals 7, 17 (1996)

24. Gelfand, I.M., Shilov, G.E.: Generalized Functions, vol. 1. Academic Press, New York (1964)

25. Haubold, H.J., Mathai, A.M.: The fractional kinetic equation and thermonuclear functions. Astrophys. Space Sci. 327 53-63 (2000)

26. Kourganoff, V.: Introduction to the Physics of Stellar Interiors. Reidel, Dordrecht (1973)

27. Samko, S.G., Kilbas, A., Marichev, O.: Fractional Integrals and Derivatives. Theory and Applications. Gordon \& Breach, New York (1990)

28. Kumar, D., Purohit, S.D., Secer, A., Atangana, A.: On generalized fractional kinetic equations involving generalized Bessel function of the first kind. Math. Probl. Eng. 2015, Article ID 289387 (2015)

29. Mittag-Leffler, G.M.: Sur la représentation analytique d'une fonction monogène: cinquième note. Acta Math. 29, 101-181 (1905)

30. Erdélyi, A., Magnus, W., Oberhettinger, F., Tricomi, F.G.: Higher Transcendental Functions, vol. 1. McGraw-Hill, New York (1953)

31. Srivastava, H.M., Gupta, K.C., Goyal, S.P.: The H-Functions of One and Two Variables with Applications. South Asian Publishers, New Delhi (1982)

32. Srivastava, H.M., Karlsson, P.W.: Multiple Gaussian Hypergeometric Series. Halsted, New York (1985) 